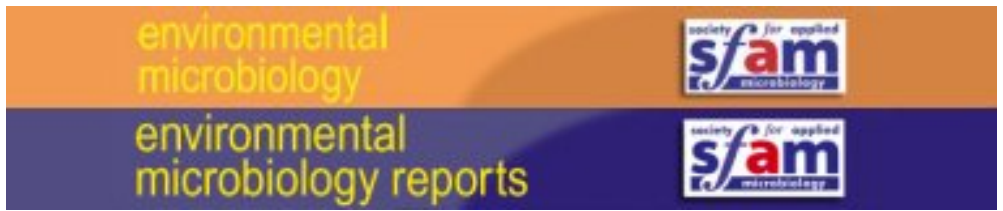

\title{
Towards meaningful scales in ecosystem microbiome research
}

\begin{tabular}{|r|l|}
\hline Journal: & Environmental Microbiology and Environmental Microbiology Reports \\
\hline Manuscript ID & EMI-2020-1477.R1 \\
\hline Journal: & Environmental Microbiology \\
\hline Manuscript Type: & EMI - Correspondance \\
\hline Date Submitted by the & n/a \\
\hline Complete List of Authors: & $\begin{array}{l}\text { Dini-Andreote, Francisco; The Pennsylvania State University - Main } \\
\text { Campus, Plant Science } \\
\text { Kowalchuk, George; Utrecht University, Ecology and Biodiversity } \\
\text { Prosser, James; University of Aberdeen, School of Biological Sciences } \\
\text { Raaijmakers, Jos; NIOO-KNAW, Microbial Ecology }\end{array}$ \\
\hline Keywords: & $\begin{array}{l}\text { species distribution, ecological patterns, microbial communities, } \\
\text { microbial ecology }\end{array}$ \\
\hline &
\end{tabular}

\section{SCHOLARONE Manuscripts}




\section{Towards meaningful scales in ecosystem microbiome research}

3 Article type. Correspondence

4 Running title. The problem of scale in ecosystem microbiomes

6 Authors. Francisco Dini-Andreote ${ }^{* 1,2}$, George A. Kowalchuk ${ }^{3}$, James I. Prosser ${ }^{4}$, Jos M. Raaijmakers ${ }^{2,5}$

8 Authors affiliation. ${ }^{1}$ Department of Plant Science \& Huck Institutes of the Life Sciences, The Pennsylvania

9 State University, University Park, PA, USA; ${ }^{2}$ Department of Microbial Ecology, Netherlands Institute of

10 Ecology (NIOO-KNAW), Wageningen, The Netherlands; ${ }^{3}$ Ecology \& Biodiversity, Institute of Environmental

11 Biology, Utrecht University, Utrecht, The Netherlands; ${ }^{4}$ School of Biological Sciences, University of

12 Aberdeen, Aberdeen, United Kingdom; ${ }^{5}$ nstitute of Biology, Leiden University, Leiden, The Netherlands

14 Keywords. species distribution; ecological patterns; communities; microbial ecology

*Corresponding author. Francisco Dini-Andreote, Department of Plant Science \& Huck Institutes of the Life

17 Sciences, The Pennsylvania State University. 220 Wartik Laboratory, University Park, State College, PA, USA,

18 16801. E-mail: andreote@psu.edu, Phone: +1 8148632188. 


\section{Originality-Significance Statement}

21 This is a critical article that articulates on the main issues to be considered in properly addressing the

22 problem of scale in ecosystem microbiome research. We aim at stimulating debate on this challenging and

23 often neglected topic. We advocate that efforts towards defining and using meaningful scales in ecosystem

24 microbiome assessments will promote study reproducibility and advance both conceptual and theoretical

25 developments. This will also contribute to redirect research priorities to enhance our mechanistic

26 understanding of taxa distributional patterns and the ecosystem functions they provide.

\section{Summary}

29 Studies of microbial communities in natural ecosystems have been generally focused on mapping patterns

30 of species and gene distributions. Although highly instrumental in expanding our understanding of

31 microbial diversity and distribution patterns, such census studies often lack a meaningful and explicit

32 definition of scale. Here, we discuss the importance of scale in environmental microbiology assessments

33 and consider how patterning ecology can be redirected towards advancing concept and theory formation

34 in ecosystem microbiome research.

\section{Introduction}

37 Increasing attention is being given to mapping 'global' and/or 'cross-continental' patterns of microbial 38 communities across ecosystems (e.g., Ladau et al., 2013; Bates et al., 2013; Tedersoo et al., 2014; Zhou et 39 al., 2016; Delgado-Baquerizo et al., 2018; Baham et al., 2018). The majority of these studies are founded 40 on the notion that cataloguing large-scale diversity and distributional patterns of microbes will improve

41 our understanding of the importance of microbiomes and specific taxon/gene abundances for predicting 42 regional and global ecosystem processes; e.g. carbon source-sink dynamics and positive or negative 
43 microbially mediated effects on global warming (Crowther et al., 2019). These studies have increased

44 knowledge of, for instance, the ubiquitous versus rare distribution of taxa and functions. However, less

45 attention has been given to assessing the reliability or value of the often-arbitrary scales used. We argue

46 that without an explicit consideration of scale and its limitations, extrapolating findings obtained from

47 limited datasets can lead to misleading information, thus hindering our ability to effectively understand

48 and predict the functional capacity of environmental microbiomes. To stimulate debate on this challenging

49 topic, we consider three main categorical issues associated with terminology, concept formation and 50 theory construction.

\section{The terminology problem}

53 The use of 'global/regional/cross-continental' and 'pattern/distribution/biogeography' terminologies has 54 been broadly used to refer to large-scale surveys of ecosystem microbiomes. It is important to realize that 55 data obtained from these assessments are derived from a limited set of samples collected at particularly 56 small scales (e.g., 1-10 $\mathrm{mL}$ of water or $<1 \mathrm{~g}$ of soil), with results later extrapolated to larger spatial scales 57 using scattered sampling designs without accounting for spatial environmental variation. Although typically 58 not stated explicitly, it is often implied that such datasets are in fact representative of all sites at that scale, 59 including non-sampled sites for which information is unavailable. In defence of this approach, it could be 60 argued that it is not feasible to perform fine-scale sampling for broad-scale studies that range from 61 thousands of kilometres to continental scales. In addition, meaningful or not, correlational outcomes are 62 often robust and sufficiently significant to justify hypothesis development and the further exploration of 63 potential underlying processes and mechanisms governing taxon distribution patterns. In fact, both of 64 these arguments suffer from the same fallacy, which is an assumption that is best illustrated by the logical 65 argument from classical scientific philosophy: "Absence of evidence is not evidence of absence". That is, 66 since it is impossible to cover at a fine-scale potential site variation across broad spatial scales (i.e., absence 
67 of evidence), whatever patterns emerge from a limited (often scattered) collection of samples will hold for

68 all missing samples within that scale (evidence of absence). Thus, as a consequence of this fundamental

69 design problem, outcomes will be biased by non-random datasets, at least to some extent, and there is no

70 explicitly defined/optimal sampling plan. Eliminating these issues - or at least diminishing their importance

71 - requires the use of proper language, the explicit definition of scale and its limitations, and proper

72 accounting for both replication and assessment of within-site variation when developing broad-scale

73 sampling designs.

\section{Concept formation}

76 The debate about patterns and scale in ecology is not new (see Chave, 2013) but these concepts remain

77 poorly developed in environmental microbiology (Ladau and Eloe-Fadrosh, 2019). As revised by Levin

78 (1992) and Chave (2013), the main objective of patterning ecology is to inform theory. Thus, it is critically

79 important that patterns have some degree of repetition and replicability if they are to have any level of

80 predictive power (MacArthur, 1972). Replication and spatially independent representative sampling are

81 therefore essential to infer both small-scale and large-scale patterns, as is the incorporation of nested

82 designs in accordance with scale to account for autocorrelation (Tedersoo, 2017). Moreover, reliable and

83 informed prediction can only be achieved through sufficient longitudinal data and/or by clear elucidation

84 of mechanisms that underlie patterns (Levin, 1992), both of which require a meaningful and explicit

85 definition of scale. Importantly, the relative influences of distinct mechanisms underpinning taxa

86 distributions are known to vary as a function of scale, so-called scale-dependency (Dini-Andreote et al.,

87 2015; Chase et al., 2018). Thus, starting with a clean slate and going from processes to patterns, and not

88 backwards, may represent a more fruitful strategy to advance mechanistic understanding of microbial

89 distributional patterns. 
91 determination of scales" (Levin, 1992). This raises a critical limitation, which is the extent to which patterns

92 or variation that emerge from spatially limited designs and lack of temporal components preclude many

93 studies from appropriate replication. In other words, are these assessments providing global patterns of

94 biological diversity or intrinsic variation within sample sets collected at sites scattered at a global scale?

95 For example, samples may be collected across a range of environmental variable(s), and these variable(s)

96 may turn out to fit correlational patterns. However, these patterns remain in the absence of evidence of

97 'how' and 'the extent at which' these variable(s) might physiologically constrain or promote the abundance

98 or activities of specific taxa. Considering $\mathrm{pH}$, which is often found to be the highest correlate to community

99 composition in terrestrial (soil) systems. Although pH can have some direct effect on microbial physiology,

100 it will also have many indirect effects that might be equally or even more important to species distributions.

101 In addition, millimetre-scale gradients in $\mathrm{O}_{2}$ in soil particles have been shown to drive community

102 divergences that can be analogous to those resulting from selection imposed by large-scale abiotic

103 variables (e.g., pH, salinity, etc.) or anthropogenic disturbances (agricultural practices, land-use conversion,

104 etc.) at a broader scale (Konopka, 2009; Vos et al., 2013). Moreover, it is worth noting that there are

105 fundamental differences when comparing aquatic and terrestrial ecosystems. Most of these differences

106 relate to the mechanisms structuring these systems and how these relate to organismal dispersal

107 limitation, spatial connectivity and environmental filtering. For example, one can argue that microbiomes

108 in surface waters in the Pacific and Atlantic oceans (similar to 'continental scales') may be more similar

109 than those collected in a single area but will differ in the photic and twilight zones (mesopelagic zone, 200-

$1101,000 \mathrm{~m})$. Furthermore, samples for soil microbiome assessments usually undergo homogenization of

111 micro-scale 'habitats' during processing. This is less of a problem in aquatic systems, in which the

112 distribution of habitat gradients varies at a larger scale (ca. 1-10 m) than in terrestrial systems (ca. 0.1-1

$113 \mathrm{~mm}$ ). Thus, attention should be given to the intrinsic characteristics of divergent systems, the scales at 
114 which biogeochemical gradients are formed and those at which ecological processes operate. There is,

115 therefore, no such thing as a 'gold standard' by which one can define an 'optimum scale' (also called

116 'characteristic scale', i.e. one that 'maximizes the ratio of deterministic information to stochastic

117 fluctuations' sensu Pascual and Levin, 1999). Rather, there is an urgent need for reproducibility with an

118 explicit consideration of scale from which patterns emerge, thus paving the way for conceptual and

119 theoretical developments.

\section{Theory construction}

122 "Theory is used to classify, interpret and predict the world around us. Without it, microbial ecology is merely 123 the accumulation of situation-bound statements that are of limited predictive ability, providing 124 microbiologists with few insights"'(Prosser et al., 2009).

125 An ecological theory is built upon a contemplative amalgamate of information that aims to explain 126 generalized patterns in nature. Most importantly, an ecological theory requires an 'explanatory surplus' 127 (Gillies, 2015), that is, the ability to describe phenomena outside of the immediate realm in which it was 128 formulated. This means that patterning microbiomes across ecosystems require a clear consideration of 129 scale that results in consistency, a level of reproducibility and integration of information, as well as 130 empirical tests and patterning validation assessments. Non-reproducible patterns are likely to result in 131 obscure noise and/or lead to an overall assessment that microbiomes are stochastically assembled; or that 132 patterns emerging from a reductionist approach are applicable at broad scales. This would reinforce 133 assumptions that predicting, monitoring and/or manipulating microbiomes are unrealistic tasks and 134 promote the argument of context-dependency and absence of realistic trackable mechanisms 135 underpinning divergence in community assemblages across divergent systems and scales. 
136 Two of the main objectives of theory are to inform applied ecology and experimental design. Patterns in

137 ecosystem microbiomes can promote theory development by providing cross-system assessments using 138 microbe-centric sampling strategies that also include appropriate measures of the local environmental 139 complexity. The emerging data, together with other macroscale data, can then be used to develop 140 validation experiments or environmental assessments that test hypotheses about potential underlying 141 ecological and evolutionary mechanisms. As a path forward, and as similarly debated in general ecology, 142 focus should be given to study systems at appropriate and pre-defined scales. This will later inform the 143 development of models that bridge different scales (Melbourne and Chesson, 2006; Holt and Chesson, 144 2016; Chesson, 2010; Chase et al., 2018), and avoid the accumulation of an anecdotal collection of datasets 145 that may confound concept formation and therefore limit, rather than advance, the development of 146 ecological theory.

\section{Recommendations}

149 This article has aimed to stimulate debate on how scale can be reliable and meaningfully defined and taken 150 into practice in ecosystem microbiome research. The arguments and recommendations are not intended 151 to discredit or demerit previous research efforts. We also do not claim to have now closed this debate nor 152 do we have the ultimate solutions. However, we provide some initial recommendations that we believe 153 will benefit future research. In brief, to promote conceptual and theory formation, studies should consider 154 the following recommendations: (1) Explicitly define scale and the extent to which it is representative and 155 relevant to potentially underlying mechanisms. This should lead to a level of reproducibility that allows for the proper elucidation and validation of underlying mechanisms and processes that govern community 157 divergences. (2) Access variability within local sites and make use of new methods to assist sampling design 158 (e.g., response-surface methodology, see Albert et al., 2010). This is critical for the development of experimental plans that enhance sample/site representativeness. Information on local variability can also 
160 be used to feed model predictions and indicate the level of uncertainty when attempting to extrapolate

161 patterns. (3) Account for inaccurate extrapolations by indicating levels of uncertainty in model predictions

162 and data visualization, as well as acknowledging the existence of sites for which information is unavailable.

163 Together, these will enhance the clarity of data presentation and promote discussion on challenges and

164 limitations associated with sampling design. (4) When similar ecosystems are surveyed across large-scale

165 gradients, studies should consider using replicable spatial designs with consistent sample sizes that

166 potentially control for autocorrelation of variables. Particularly in the case of autocorrelation and

167 structured data, models using k-fold cross-validation on geographically-partitioned datasets have been

168 successfully used in species distribution modelling and has led to much more generalizable model fits (e.g.

169 Roberts et al., 2017). (5) Since correlational outcomes can be meaningfully applied to generate and test

170 hypotheses empirically, value should be given to studies that develop prospective experimental designs

171 aimed at falsifying putative mechanisms. This represents a challenging yet elegant way towards advancing

172 this fast-moving field of science. In conclusion, despite the various ways in which scale and spatiotemporal

173 variability can subvert our interpretation of microbial taxa and gene distributional patterns (also see

174 Armitage and Jones, 2019), we hope new studies in 'global/regional/cross-continental' microbiome

175 assessments will take these constructive suggestions into account. Collectively, these will help to orient

176 new experimental designs and enhance precision in data analysis, interpretation and communication. Thus,

177 paving the way for a more realistic appreciation of the advances and impacts ecosystem microbiome 178 surveys truly provide. 
The authors declare no conflict of interest.

\section{References}

Albert, C.H., Yoccoz, N.G., Edwards, T.C., Graham, C.H., Zimmermann, N.E., Thuiller, W. (2010). Sampling in ecology and evolution - bridging the gap between theory and practice. Ecography 33: 1028-1037.

Armitage, D.W., and Jones, S.E. (2019). How sample heterogeneity can obscure the signal of microbial interactions. ISME J 13: 2639-2646.

Bahram, M., Hildebrand, F., Forslund, S.K., Anderson, J.L., Soudzilovskaia, N.A., Bodegom, P.M., et al. (2018). Structure and function of the global topsoil microbiome. Nature 560: 233-237. biogeography of highly diverse protistan communities in soil. ISME J 7: 652-659.

Chase, J.M., McGill, B.J., McGlinn, D.J., May, F., Blowes, S.A., Xiao, X., et al. (2018). Embracing scale-dependence to achieve a deeper understanding of biodiversity and its change across communities. Ecol Lett 21: 1737-1751.

Chave, J. (2013). The problem of pattern and scale in ecology: what have we learned in 20 years? Ecol Lett 16: 4-16.

Chesson, P. (2010). Scale transition theory with special reference to species coexistence in a variable environment. J Biol Dynam 3: 149-163.

Crowther, T.W., van den Hoogen, J., Wan, J., Mayes, M.A., Keiser, A.D., Mo, L., et al. (2019). The global soil community and its influence on biogeochemistry. Science 365: 772.

Delgado-Baquerizo, M., Oliverio, A.M., Brewer, T.E., Benavent-González, A., Eldridge, D.J., Bardgett, R.D., et al. (2018). A global atlas of the dominant bacteria found in soil. Science 359: 320-325. 
203

Dini-Andreote, F., Stegen, J.C., van Elsas, J.D., Salles, J.F. (2015). Disentangling mechanisms that mediate the balance between stochastic and deterministic processes in microbial succession. Proc Natl Acad Sci U S A 112: E1326-E1332.

Gillies, D.A. (2015). Non-Bayesian confirmation theory, and the principle of explanatory surplus. Philos Sci 1988: 2 .

Holt, G., and Chesson, P. (2016). Scale-dependent community theory for streams and other linear habitats. Am Nat 188: E59-E73.

Konopka, A. (2009). What is microbial community ecology? ISME J 3: 1223-1230.

Ladau, J., and Eloe-Fadrosh, E.A. (2019). Spatial, temporal, and phylogenetic scales of microbial ecology. Trends Microbiol 27: 662-669.

Ladau, J., Sharpton, T.J., Finucane, M.M., Jospin, G., Kembel, S.W., O'Dwyer, J., et al. (2013). Global marine bacterial diversity peaks at high latitudes in winter. ISME J 7: 1669-1677.

Levin, A.S. (1992). The problem of pattern and scale in ecology. Ecology 73: 1943-1967.

MacArthur, R.H. (1972) Geographical ecology: patterns in the distribution of species. New York, USA: Harper and Row.

Melbourne, B.A., and Chesson, P. (2006). The scale transition: scaling up population dynamics with field data. Ecology 87: 1478-1488.

Pascual, M., and Levin, S.A. (1999). From individuals to population densities: searching for the intermediate scale of nontrivial determinism. Ecology 80: 2225-2236.

Prosser, J.I., Bohannan, B.J.M., Curtis, T.P., Ellis, R.J., Firestone, M.K., Freckleton, R.P., et al. (2007). The role of ecological theory in microbial ecology. Nat Rev Microbiol 5: 384-392.

Roberts, D.R., Bahn, V., Ciuti, S., Boyce, M.S., Elith, J., Guillera-Arroita, G., et al. (2017). Cross-validation strategies for data with temporal, spatial, hierarchical, or phylogenetic structure. Ecography 40: 913929. 
227 Tedersoo, L. (2017). Analytical flaws in a continental-scale forest soil microbial diversity study. Nat Commun 228 8: 15572

229 Tedersoo, L., Bahram M., Põlme, S., Kõljalg, U., Yorou, N.S., Wijesundera, R., et al. (2014). Global diversity 230 and geography of soil fungi. Science 346: 1256688.

231 Vos, M., Wolf, A.B., Jennings, S.J., Kowalchuk, G.A. (2013). Micro-scale determinants of bacterial diversity 232 in soil. FEMS Microbiol Rev 37: 936-954.

233 Zhou, J., Deng, Y., Shen, L., Wen, C., Yan, Q., Ning, D., et al. (2016). Temperature mediates continental-scale 234 diversity of microbes in forest soils. Nat Commun 7: 12083. 


\section{Towards meaningful scales in ecosystem microbiome research}

2

3 Article type. Correspondence

$4 \quad$ Running title. The problem of scale in ecosystem microbiomes

5

6 Authors. Francisco Dini-Andreote ${ }^{* 1,2}$, George A. Kowalchuk ${ }^{3}$, James I. Prosser ${ }^{4}$, Jos M. Raaijmakers ${ }^{2,5}$

8 Authors affiliation. ${ }^{1}$ Department of Plant Science \& Huck Institutes of the Life Sciences, The Pennsylvania

9 State University, University Park, PA, USA; ${ }^{2}$ Department of Microbial Ecology, Netherlands Institute of

10 Ecology (NIOO-KNAW), Wageningen, The Netherlands; ${ }^{3}$ Ecology \& Biodiversity, Institute of Environmental

11 Biology, Utrecht University, Utrecht, The Netherlands; ${ }^{4}$ School of Biological Sciences, University of

12 Aberdeen, Aberdeen, United Kingdom; ${ }^{5}$ Institute of Biology, Leiden University, Leiden, The Netherlands

14 Keywords. species distribution; ecological patterns; communities; microbial ecology

*Corresponding author. Francisco Dini-Andreote, Department of Plant Science \& Huck Institutes of the Life

17 Sciences, The Pennsylvania State University. 220 Wartik Laboratory, University Park, State College, PA, USA,

18 16801. E-mail: andreote@psu.edu, Phone: +1 8148632188.

19 
21 This is a critical article that articulates on the main issues to be considered in properly addressing the

22 problem of scale in ecosystem microbiome research. We aim at stimulating debate on this challenging and

23 often neglected topic. We advocate that efforts towards defining and using meaningful scales in ecosystem

24 microbiome assessments will promote study reproducibility and advance both conceptual and theoretical

25 developments. This will also contribute to redirect research priorities to enhance our mechanistic

26 understanding of taxa distributional patterns and the ecosystem functions they provide.

\section{Summary}

29 Studies of microbial communities in natural ecosystems have been generally focused on mapping patterns

30 of species and gene distributions. Although highly instrumental in expanding our understanding of

31 microbial diversity and distribution patterns, such census studies often lack a meaningful and explicit

32 definition of scale. Here, we discuss the importance of scale in environmental microbiology assessments

33 and consider how patterning ecology can be redirected towards advancing concept and theory formation

34 in ecosystem microbiome research.

\section{Introduction}

37 Increasing attention is being given to mapping 'global' and/or 'cross-continental' patterns of microbial 38 communities across ecosystems (e.g., Ladau et al., 2013; Bates et al., 2013; Tedersoo et al., 2014; Zhou et 39 al., 2016; Delgado-Baquerizo et al., 2018; Baham et al., 2018). The majority of these studies are founded 40 on the notion that cataloguing large-scale diversity and distributional patterns of microbes will improve

41 our understanding of the importance of microbiomes and specific taxon/gene abundances for predicting 42 regional and global ecosystem processes; e.g. carbon source-sink dynamics and positive or negative 
43 microbially mediated effects on global warming (Crowther et al., 2019). These studies have increased

44 knowledge of, for instance, the ubiquitous versus rare distribution of taxa and functions. However, less

45 attention has been given to assessing the reliability or value of the often-arbitrary scales used. We argue

46 that without an explicit consideration of scale and its limitations, extrapolating findings obtained from

47 limited datasets can lead to misleading information, thus hindering our ability to effectively understand

48 and predict the functional capacity of environmental microbiomes. To stimulate debate on this challenging

49 topic, we consider three main categorical issues associated with terminology ${ }_{2}$ and-concept formation and

50 theory formationconstruction.

The terminology problem

53 The use of 'global/regional/cross-continental' and 'pattern/distribution/biogeography' terminologies has

54 been broadly used to refer to large-scale surveys of ecosystem microbiomes. It is important to realize that

55 data obtained from these assessments are derived from a limited set of samples collected at particularly

56 small scales (e.g., 1-10 mL of water or $<1 \mathrm{~g}$ of soil), with results later extrapolated to larger spatial scales

57 using scattered sampling designs without accounting for spatial environmental variation. Although typically

58 not stated explicitly, it is often implied that such datasets are in fact representative of all sites at that scale,

59 including non-sampled sites for which information is unavailable. In defence of this approach, it could be

60 argued that it is not feasible to perform fine-scale sampling for broad-scale studies that range from

61 thousands of kilometres to continental scales. In addition, meaningful or not, correlational outcomes are

62 often robust and sufficiently significant to justify hypothesis development and the further exploration of

63 potential underlying processes and mechanisms governing taxon distribution patterns. In fact, both of

64 these arguments suffer from the same fallacy, which is an assumption that is best illustrated by the logical

65 argument from classical scientific philosophy: "Absence of evidence is not evidence of absence". That is,

66 since it is impossible to cover at a fine-scale potential site variation across broad spatial scales (i.e., absence 
67 of evidence), whatever patterns emerge from a limited (often scattered) collection of samples will hold for

68 all missing samples within that scale (evidence of absence). Thus, as a consequence of this fundamental

69 design problem, outcomes will be biased by non-random datasets, at least to some extent, and there is no

70 explicitly defined/optimal sampling plan. Eliminating these issues - or at least diminishing their importance

71 - requires the use of proper language, the explicit definition of scale and its limitations, and proper

72 accounting for both replication and assessment of within-site variation when developing broad-scale

73 sampling designs.

\section{Concept formation}

76 The debate about patterns and scale in ecology is not new (see Chave, 2013) but these concepts remain

77 poorly developed in environmental microbiology (Ladau and Eloe-Fadrosh, 2019). As revised by Levin

78 (1992) and Chave (2013), the main objective of patterning ecology is to inform theory. Thus, it is critically

79 important that patterns have some degree of repetition and replicability if they are to have any level of

80 predictive power (MacArthur, 1972). Replication and spatially independent representative sampling are

81 therefore essential to infer both small-scale and large-scale patterns, as is the incorporation of nested

82 designs in accordance with scale to account for autocorrelation (Tedersoo, 2017). Moreover, reliable and

83 informed prediction can only be achieved through sufficient longitudinal data and/or by clear elucidation

84 of mechanisms that underlie patterns (Levin, 1992), both of which require a meaningful and explicit

85 definition of scale. Importantly, the relative influences of distinct mechanisms underpinning taxa

86 distributions are known to vary as a function of scale, so-called scale-dependency (Dini-Andreote et al.,

87 2015; Chase et al., 2018). Thus, starting with a clean slate and going from processes to patterns, and not

88 backwards, may represent a more fruitful strategy to advance mechanistic understanding of microbial

89 distributional patterns. 
90 "The description of patterns is the description of variation, and the quantification of variation requires the

91 determination of scales" (Levin, 1992). This raises a critical limitation, which is the extent to which patterns

92 or variation that emerge from spatially limited designs and lack of temporal components preclude many

93 studies from appropriate replication. In other words, are these assessments providing global patterns of

94 biological diversity or intrinsic variation within sample sets collected at sites scattered at a global scale?

95 For example, samples may be collected across a range of environmental variable(s), and these variable(s)

96 may turn out to fit correlational patterns. However, these patterns remain in the absence of evidence of

97 'how' and 'the extent at which' these variable(s) might physiologically constrain or promote the abundance

98 or activities of specific taxa. Considering $\mathrm{pH}$, which is often found to be the highest correlate to community

99 composition in terrestrial (soil) systems. Although pH can have some direct effect on microbial physiology,

100 it will also have many indirect effects that might be equally or even more important to species distributions.

101 In addition, millimetre-scale gradients in $\mathrm{O}_{2}$ in soil particles have been shown to drive community 102 divergences that can be analogous to those resulting from selection imposed by large-scale abiotic 103 variables (e.g., pH, salinity, etc.) or anthropogenic disturbances (agricultural practices, land-use conversion, 104 etc.) at a broader scale (Konopka, 2009; Vos et al., 2013). Moreover, it is worth noting that there are 105 fundamental differences when comparing aquatic and terrestrial ecosystems. Most of these differences 106 relate to the mechanisms structuring these systems and how these relate to organismal dispersal 107 limitation, spatial connectivity and environmental filtering. For example, one can argue that microbiomes 108 in surface waters in the Pacific and Atlantic oceans (similar to 'continental scales') may be more similar 109 than those collected in a single area but will differ in the photic and twilight zones (mesopelagic zone, 200$1101,000 \mathrm{~m})$. Furthermore, samples for soil microbiome assessments usually undergo homogenization of 111 micro-scale 'habitats' during processing. This is less of a problem in aquatic systems, in which the 112 distribution of habitat gradients varies at a larger scale (ca. 1-10 m) than in terrestrial systems (ca. 0.1-1 $113 \mathrm{~mm})$. Thus, attention should be given to the intrinsic characteristics of divergent systems, the scales at 
114 which biogeochemical gradients are formed and those at which ecological processes operate. There is,

115 therefore, no such thing as a 'gold standard' by which one can define an 'optimum scale' (also called

116 'characteristic scale', i.e. one that 'maximizes the ratio of deterministic information to stochastic

117 fluctuations' sensu Pascual and Levin, 1999). Rather, there is an urgent need for reproducibility with an

118 explicit consideration of scale at which mechanisms operate and from which patterns emerge, thus paving

119 the way for conceptual and theoretical developments.

\section{Theory construction}

122 "Theory is used to classify, interpret and predict the world around us. Without it, microbial ecology is merely 123 the accumulation of situation-bound statements that are of limited predictive ability, providing 124 microbiologists with few insights"'(Prosser et al., 2009).

125 An ecological theory is built upon a contemplative amalgamate of information that aims to explain 126 generalized patterns in nature. Most importantly, an ecological theory requires an 'explanatory surplus' 127 (Gillies, 2015), that is, the ability to describe phenomena outside of the immediate realm in which it was 128 formulated. This means that patterning microbiomes across ecosystems require a clear consideration of 129 scale that results in consistency, a level of reproducibility and integration of information, as well as 130 empirical tests and patterning validation assessments. Non-reproducible patterns are likely to result in 131 obscure noise and/or lead to an overall assessment that microbiomes are stochastically assembled; or that 132 patterns emerging from a reductionist approach are applicable at broad scales. This would reinforce 133 assumptions that predicting, monitoring and/or manipulating microbiomes are unrealistic tasks and 134 promote the argument of context-dependency and absence of realistic trackable mechanisms 135 underpinning divergence in community assemblages across divergent systems and scales. 
136 Two of the main objectives of theory are to inform applied ecology and experimental design. Patterns in

137 ecosystem microbiomes can promote theory development by providing cross-system assessments using 138 microbe-centric sampling strategies that also include appropriate measures of the local environmental 139 complexity. The emerging data, together with other macroscale data, can then be used to develop 140 validation experiments or environmental assessments that test hypotheses about potential underlying 141 ecological and evolutionary mechanisms. As a path forward, and as similarly debated in general ecology, 142 focus should be given to study systems at appropriate and pre-defined scales. This will later inform the 143 development of models that bridge different scales (Melbourne and Chesson, 2006; Holt and Chesson, 144 2016; Chesson, 2010; Chase et al., 2018), and avoid the accumulation of an anecdotal collection of datasets 145 that may confound concept formation and therefore limit, rather than advance, the development of 146 ecological theory.

149 Recommendations-moving forward

150 This article has aimed to stimulate debate on how scale can be reliable and meaningfully defined and taken

151 into practice in ecosystem microbiome research. The arguments and recommendations are not intended 152 to discredit or demerit previous research efforts. We also do not claim to have now closed this debate nor 153 do we have the ultimate solutions. However, we provide some initial recommendations that we believe 154 will benefit future research. In brief, to promote conceptual and theory formation, studies should consider

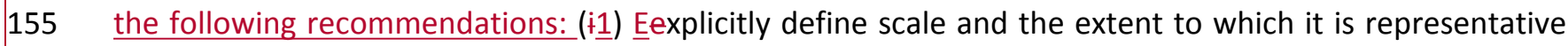
156 and relevant to potentially underlying mechanisms. This should then-lead to (ii)-a level of reproducibility 157 that (iii)-allows for the proper elucidation and validation of underlying mechanisms and processes that 158 govern community divergences. (2) Access variability within local sites and make use of new We suggest 
159 that accessing variability within local sites and introducing new-methods to assist sampling design (for 160 instancee.g., response-surface methodology, see Albert et al., 2010). This is critical for -can lead-the 161 development of experimental plans that enhancesthat enhance sample/site representativeness. 162 Information on local variability can also be used to feed model predictions and indicate the level of 163 uncertainty when attempting to extrapolate patterns. (3) Accounting Account for inaccurate extrapolations 164 by indicating levels of uncertainty in model predictions and data visualization, as well as acknowledging the 165 existence of sites for which information is unavailable. Together, these, ean-will enhance the clarity of data

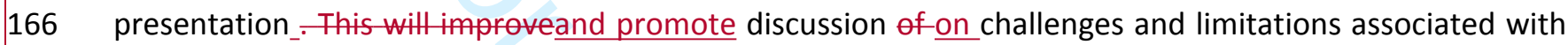
167 sampling design, thus avoiding overarching assumptions and statements and the noise these may echo. (4) 168 Moreover, when similar ecosystems are surveyed across large-scale gradients, the $\underline{\text { studies should }}$ 169 consider use using of replicable spatial designs with consistent sample sizes that potentially control for 170 autocorrelation of variables-an be beneficial. It is worth noting, pParticularly in the case of autocorrelation 171 and structured data, that-models using k-fold cross-validation on geographically-partitioned datasets have 172 been successfully used in species distribution modelling and has led to much more generalizable model fits 173 (e.g. Roberts et al., 2017). (5) Since correlational outcomes can be meaningfully applied to generate and 174 test hypotheses empirically, value should be given to studies that develop prospective experimental 175 designs aimed at falsifying putative mechanisms. This represents a challenging yet elegant way towards 176 advancing this fast-moving field of science.t_ In conclusion, despite the various ways in which scale and 177 spatiotemporal variability can subvert our interpretation of microbial taxa and gene distributional patterns 178 (also see Armitage and Jones, 2019), we hope new studies in 'global/regional/cross-continental' 179 microbiome assessments will take into-account these constructive suggestions into account. Collectively, 180 these will help to orient new experimental designs and enhance precision in data analysis, interpretation 181 and communication. Thus, paving the way for a more realistic appreciation of the advances and impacts 182 ecosystem microbiome surveys truly provide.- 
183 Finally, since correlational outcomes can be meaningfully used to generate and test hypotheses empirically, 184 switching gears towards developing prospective experimental designs for falsifying putative mechanisms 185 represents another elegant way towards advancing this fast-moving field of science. 


\section{Conflict if interests}

187 The authors declare no conflict of interest.

References

Albert, C.H., Yoccoz, N.G., Edwards, T.C., Graham, C.H., Zimmermann, N.E., Thuiller, W. (2010). Sampling in ecology and evolution - bridging the gap between theory and practice. Ecography 33: 1028-1037.

Armitage, D.W., and Jones, S.E. (2019). How sample heterogeneity can obscure the signal of microbial interactions. ISME J 13: 2639-2646.

Bahram, M., Hildebrand, F., Forslund, S.K., Anderson, J.L., Soudzilovskaia, N.A., Bodegom, P.M., et al. (2018). Structure and function of the global topsoil microbiome. Nature 560: 233-237.

Bates, S.T., Clemente, J.C., Flores, G.E., Walters, W.A., Parfrey, L.W., Knight, R., et al. (2013). Global biogeography of highly diverse protistan communities in soil. ISME J 7: 652-659.

Chase, J.M., McGill, B.J., McGlinn, D.J., May, F., Blowes, S.A., Xiao, X., et al. (2018). Embracing scale-dependence to achieve a deeper understanding of biodiversity and its change across communities. Ecol Lett 21: 1737-1751.

Chave, J. (2013). The problem of pattern and scale in ecology: what have we learned in 20 years? Ecol Lett 16: 4-16.

Chesson, P. (2010). Scale transition theory with special reference to species coexistence in a variable environment. J Biol Dynam 3: 149-163.

Crowther, T.W., van den Hoogen, J., Wan, J., Mayes, M.A., Keiser, A.D., Mo, L., et al. (2019). The global soil community and its influence on biogeochemistry. Science 365: 772.

Delgado-Baquerizo, M., Oliverio, A.M., Brewer, T.E., Benavent-González, A., Eldridge, D.J., Bardgett, R.D., 208 et al. (2018). A global atlas of the dominant bacteria found in soil. Science 359: 320-325. 
209

210

211

212

213

214

215

216

217

218

219

220

221

222

223

224

225

226

227

228

229

230

231

232

Dini-Andreote, F., Stegen, J.C., van Elsas, J.D., Salles, J.F. (2015). Disentangling mechanisms that mediate the balance between stochastic and deterministic processes in microbial succession. Proc Natl Acad Sci US A 112: E1326-E1332.

Gillies, D.A. (2015). Non-Bayesian confirmation theory, and the principle of explanatory surplus. Philos Sci 1988: 2.

Holt, G., and Chesson, P. (2016). Scale-dependent community theory for streams and other linear habitats. Am Nat 188: E59-E73.

Konopka, A. (2009). What is microbial community ecology? ISME J 3: 1223-1230.

Ladau, J., and Eloe-Fadrosh, E.A. (2019). Spatial, temporal, and phylogenetic scales of microbial ecology. Trends Microbiol 27: 662-669.

Ladau, J., Sharpton, T.J., Finucane, M.M., Jospin, G., Kembel, S.W., O'Dwyer, J., et al. (2013). Global marine bacterial diversity peaks at high latitudes in winter. ISME J 7: 1669-1677.

Levin, A.S. (1992). The problem of pattern and scale in ecology. Ecology 73: 1943-1967.

MacArthur, R.H. (1972) Geographical ecology: patterns in the distribution of species. New York, USA: Harper and Row.

Melbourne, B.A., and Chesson, P. (2006). The scale transition: scaling up population dynamics with field data. Ecology 87: 1478-1488.

Pascual, M., and Levin, S.A. (1999). From individuals to population densities: searching for the intermediate scale of nontrivial determinism. Ecology 80: 2225-2236.

Prosser, J.I., Bohannan, B.J.M., Curtis, T.P., Ellis, R.J., Firestone, M.K., Freckleton, R.P., et al. (2007). The role of ecological theory in microbial ecology. Nat Rev Microbiol 5: 384-392.

Roberts, D.R., Bahn, V., Ciuti, S., Boyce, M.S., Elith, J., Guillera-Arroita, G., et al. (2017). Cross-validation strategies for data with temporal, spatial, hierarchical, or phylogenetic structure. Ecography 40: 913929. 
233 Tedersoo, L. (2017). Analytical flaws in a continental-scale forest soil microbial diversity study. Nat Commun 234 8: 15572

235 Tedersoo, L., Bahram M., Põlme, S., Kõljalg, U., Yorou, N.S., Wijesundera, R., et al. (2014). Global diversity 236 and geography of soil fungi. Science 346: 1256688.

237 Vos, M., Wolf, A.B., Jennings, S.J., Kowalchuk, G.A. (2013). Micro-scale determinants of bacterial diversity 238 in soil. FEMS Microbiol Rev 37: 936-954.

239 Zhou, J., Deng, Y., Shen, L., Wen, C., Yan, Q., Ning, D., et al. (2016). Temperature mediates continental-scale 240 diversity of microbes in forest soils. Nat Commun 7: 12083. 